\title{
Shape Palindromes: Analysis of Intrinsic Symmetries in 2D Articulated Shapes
}

\author{
Amit Hooda ${ }^{1}$, Michael M. Bronstein ${ }^{2}$, Alexander M. Bronstein ${ }^{3}$, and \\ Radu P. Horaud ${ }^{4}$ \\ 1 Indian Institute of Technology Delhi, India \\ 2 Inst. of Computational Science, Faculty of Informatics, \\ Università della Svizzera Italiana, Lugano, Switzerland \\ 3 Dept. of Electrical Engineering, Tel Aviv University, Israel \\ 4 INRIA Grenoble Rhône-Alpes, France
}

\begin{abstract}
Analysis of intrinsic symmetries of non-rigid and articulated shapes is an important problem in pattern recognition with numerous applications ranging from medicine to computational aesthetics. Considering articulated planar shapes as closed curves, we show how to represent their extrinsic and intrinsic symmetries as self-similarities of local descriptor sequences, which in turn have simple interpretation in the frequency domain. The problem of symmetry detection and analysis thus boils down to analysis of descriptor sequence patterns. For that purpose, we show two efficient computational methods: one based on Fourier analysis, and another on dynamic programming. Metaphorically, the later can be compared to finding palindromes in text sequences.
\end{abstract}

\section{Introduction}

Symmetry and self-similarity are frequently encountered in natural and manmade objects at all scales from macro to nano [30]. Because of the relation of symmetry to redundancy of geometric data, the knowledge of the symmetries a shape possesses can be instrumental for its compression, completion, and superresolution [16]. Many objects that are normally symmetric manifest symmetry breaking as a testimony of some anomaly or abnormal behavior. Therefore, detection of symmetry and asymmetry arises in many practical problems, including applications in medicine, aesthetics, and crystallography. Knowledge of shape symmetries as a prior has been also exploited in shape reconstruction [25], segmentation [24], face detection, recognition, and feature extraction [19].

In pattern recognition and computer vision literature, the problem of symmetry detection was studied mainly in images [15], two-dimensional [31, 2, 1] and three-dimensional shapes $[27,10,17]$. A wide spectrum of methods employed for this purpose includes approaches based on dual spaces [7], genetic algorithms [9], moments [5], pair matching [13,6], and local shape descriptors [33]. For an up-to-date overview, the reader is referred to the survey article of Liu et al. [12]. Traditionally, symmetries are considered as extrinsic geometric properties of shapes that are related to the way the shape is represented in the Euclidean 
space. From such a perspective, symmetry is synonymous to invariance to a certain set of global isometric (distance-preserving) transformation of the Euclidean space (a composition of rotation, reflection, and translation). Though adequate for rigid shapes, such a point of view is inappropriate for non-rigid or deformable ones. Due to the deformations such shapes can undergo, the extrinsic symmetries may be lost, while intrinsically the shape still remains symmetric. In [22], Raviv et al. introduced the notion of intrinsic symmetry, defined as a self-isometry of the shape with respect to some intrinsic (e.g., geodesic or diffusion) metric. Such a definition does not make any use of the embedding Euclidean space in which the shape resides and is reduced to the standard notion of an extrinsic symmetry if the Euclidean metric is used. Computationally, such distance-preserving automorphisms can be found using the Gromov-Hausdorff framework, in which an initial set of candidate symmetries is detected using a branch-and-bound algorithm $[22,23]$.

Ovsjanikov et al. [20] observed that simple eigenfunctions of the LaplaceBeltrami operator of a shape are invariant, up to a sign change, to intrinsic reflection symmetries with respect to a certain class of diffusion metrics. Consequently, reflection symmetries can be represented as sign sequences under which the corresponding eigenfunctions remain invariant, which provides for a simple algorithm for reflection symmetry detection. Another parametrization of intrinsic symmetries was proposed in [26], who noted that the self-isometry group of shapes with simple (disk- or sphere-like) topology is contained in the lowdimensional Möbius group. Detection of local self-similarity was considered in [18]; the related problem of partial symmetry detection was addressed in [17, $32,23]$. Finally, detection of symmetries can be considered as a particular case of the more general self-similar structure detection. Recent works focused on detecting repeating grid-like structures [21] or Euclidean structural redundancy in 3D data [3], as well as intrinsic self-similarity [16].

The main contribution of this paper is a way of representing 2D shape symmetries as one-dimensional structures by using invariant local descriptors. This way, the symmetry of the shape (both extrinsic and intrinsic) is manifested in specific self-similarity of the associated descriptor sequence. In the case of articulated shapes, deformations of non-rigid joints are manifested as insertions/deletions in the descriptor sequence. Such self-similarity is preserved under non-rigid deformations and can be efficiently detected using Fourier analysis or dynamic programming gapped sequence alignment algorithms used in the field of bioinformatics [29]. Our approach allows detection and classification of both extrinsic and intrinsic symmetries of connected $2 \mathrm{D}$ shapes, and is partially inspired by shape matching by means of dynamic programming [8] and the recent paper of Bruckstein and Shaked on symmetric shape partitioning [4].

\section{Model}

Let us be given a simply connected shape modeled as a closed simple planar curve $S$. The curve is parametrized as $S:[0, L] \rightarrow \mathbb{R}^{2}$. The length of the curve is given 
by $\ell(S)=\int_{0}^{L}\left\|\frac{d}{d t} S(t)\right\| d t$. In the following, we assume arclength parametrization, such that $\left\|\frac{d}{d t} S(t)\right\|=1$ and thus $L=\ell(S)$.

Articulations. The shape is called articulated [11] if it can be represented as a collection of rigid parts $S_{1}, \ldots, S_{p}$ connected by non-rigid joints $J_{1}, \ldots, J_{q}$, such that $S=\bigcup_{i=1}^{p} S_{i} \cup \bigcup_{k=1}^{q} J_{k}$. We further assume that the rigid parts $S_{i}$ are parametrized over $T_{R, i} \subset[0, L]$ (i.e., $S_{i}=S\left(T_{R, i}\right)$ ) and the joints $J_{k}$ are parametrized over $T_{J, k} \subset[0, L]$ (i.e., $J_{k}=S\left(T_{J, k}\right)$ ). Let us denote $T_{R}=$ $\bigcup_{i} T_{R, i} \subseteq[0, L]$ and $T_{J}=\bigcup_{k} T_{J, k} \subseteq[0, L]$. An articulation $S^{\prime}=\mathbf{A} S$ is obtained by applying planar rigid transformations $\mathbf{R}_{i}$ (rotations and translations) to the rigid parts, and non-rigid transformations $\mathbf{T}_{k}$ to the joints, $\mathbf{A} S=\bigcup_{i=1}^{p} \mathbf{R}_{i} S_{i} \cup$ $\bigcup_{k=1}^{q} \mathbf{T}_{k} J_{k}$. Thus, the articulation can be represented as $\mathbf{A}=\left\{\mathbf{R}_{i}, \mathbf{T}_{k}\right\}$. Obviously, rigid transformations do not change the length of the parts, hence, $\ell\left(\mathbf{R}_{i} S_{i}\right)=\ell\left(S_{i}\right)$ for $i=1, \ldots, p$.

Extrinsic Symmetry. Planar transformations preserving Euclidean distances are called isometries and include rotations, translations, reflections, and their compositions. The set Iso $\left(\mathbb{R}^{2}\right)$ of Euclidean isometries together with function composition operator forms the isometry group of $\mathbb{R}^{2}$. The $\operatorname{subgroup} \operatorname{Sym}(S)$ $\subset \mathrm{Iso}\left(\mathbb{R}^{2}\right)$ Euclidean isometries to which the shape $S$ is invariant (i.e. $\mathbf{R} S=S$ for all $\mathbf{R} \in \operatorname{Sym}(S)$ ) is called the extrinsic symmetry group of $S$. Elements of the group from which the entire group can be produced are called the group generators.

The structure of the symmetry group tells us "in which way" the object is symmetric. The trivial case is the $C_{1}=\{\mathrm{id}\}$ group, containing only the identity transformation (such shapes are usually called asymmetric). Rotation symmetry is described by a cyclic group $C_{n}$, generated by the rotations transformation around a fixed center by the angle $2 \pi / n$. Bilateral symmetry is described by the dihedral group $D_{1}$, consisting of an identity and a single reflection around a symmetry axis. More general dihedral symmetry of order $n$ (described by the semidirect product group $D_{n}=C_{n} \times C_{2}$ ) is generated by a rotation around a fixed center by the angle $2 \pi / n$ and a reflection around an axis passing through the center.

Intrinsic Symmetry. An articulation may break the extrinsic symmetry, such that the resulting shape is no more symmetric in the above sense. Yet, considering the intrinsic geometry of the shape and the group of isometries preserving this geometry, one can define a broader notion of intrinsic symmetry, which will hold in this case [22]. In our formulation, the shape $S$ is said to be intrinsically symmetric if there exists an articulation $\mathbf{A}$ such that $\mathbf{A} S$ is extrinsically symmetric (i.e., has a non-trivial extrinsic symmetry group). In other words, $S$ can be "symmetrized" by means of an articulation $\mathbf{A}=\left\{\mathbf{R}_{i}, \mathbf{T}_{k}\right\}$, from which it follows that $\mathbf{R} \bigcup_{i=1}^{p} \mathbf{R}_{i} S_{i}=\bigcup_{i=1}^{p} \mathbf{R}_{i} S_{i}$, where $\mathbf{R} \in \operatorname{Iso}\left(\mathbb{R}^{2}\right)$.

\section{Symmetry Analysis}

An important problem in shape analysis is, given a shape $S$, to automatically determine its symmetry group. This problem is often referred to as symmetry 
analysis, classification, or detection. In this section, we present a method for symmetry classification based on the analysis of corresponding shape descriptors. We first show a representation of extrinsic symmetry groups in the descriptor sequence and its Fourier transform domain. Next, we extend our analysis to the intrinsic case using the articulation model.

At each point on the shape contour $S(t)$, we define a scalar or vector descriptor $a_{S}(t)$, which is local and invariant to shape isometries. A simple example of such a descriptor is the curvature $\kappa(t)$ Since the curvature involves second-order derivatives, it is sensitive to noise (more generally, all differential invariants tend to be sensitive to noise). Alternatively, we can use as $a_{S}$ the integral invariant proposed in [14], defined as $I(t)=\int_{S}\left\|S(t)-S\left(t^{\prime}\right)\right\| q\left(t, t^{\prime}\right) d t^{\prime}$, where $\|\cdot\|$ denotes the Euclidean distance, and $q$ is a local kernel decreasing with the distance used to localize the descriptor. Such a descriptor is also invariant to Euclidean isometries. Using any local descriptor representation $a_{S}(t)$, the curve can be considered as a continuous sequence over $\mathbb{R} \bmod \ell(S)$. Assuming that the descriptor is invariant under rigid transformations, the descriptor sequences at the corresponding points of an articulated shape coincide.

The key idea of the approach presented in this paper is to identify the planar shape transformations with transformations of the descriptor sequences. In other words, given a symmetric shape satisfying $\mathbf{R} S=S$, the shape invariance under the symmetry transformation $\mathbf{R}$ can be related to descriptor sequence invariance, $a_{S}(t)=\left(a_{S} \circ \tau\right)(t)$, under a re-parametrization transformation $\tau$.

\subsection{Extrinsic Symmetry Characterization}

Rotation Symmetry. The action of an element of the group $C_{n}$ (rotation by $2 \pi k / n)$ is manifested as $a_{S}(t)=a_{S}(k L / n+t \bmod L)$. Thus, the descriptor sequence of a $C_{n}$-symmetric shape is $L / n$-periodic. Looking at the Fourier transform $\hat{a}_{S}(\omega)=\int_{0}^{L} a_{S}(t) e^{-2 \pi j \omega t} d t$ of the descriptor sequence, the periodicity of $a_{S}(t)$ is manifested in $\hat{a}_{S}(\omega)$ being discrete with step $2 \pi n / L$.

Dihedral Symmetry. In the simplest case of bilateral $\left(D_{1}\right)$ symmetry, the action of a reflection transformation is manifested as $a_{S}\left(t_{0}+t \bmod L\right)=a_{S}\left(t_{0}-\right.$ $t \bmod L)$, where $S\left(t_{0}\right)$ is a point on the symmetry axis that is mapped to itself. We shall refer to this point as center of reflection symmetry (note that the point $t_{0}+L / 2$ is also a center; we shall call such pairs conjugate centers). Thus, the descriptor sequence of a bilaterally-symmetric shape is an even function about the point $t_{0}$. Consequently, $a_{S}\left(t_{0}+t \bmod L\right)$ is an even function about origin, which means that its Fourier transform is real and even. By translation property of Fourier transform, we get $\hat{a}_{S}(\omega)=\mathcal{F}\left\{a_{S}\left(t_{0}+t \bmod L\right)\right\} e^{-2 \pi \iota t_{0} \omega}$, which is the polar representation of $\hat{a}_{S}$. The phase encodes the position of the reflection point $t_{0}$; it varies linearly with $\omega$ and $t_{0}$ is the slope of the line.

More generally, a dihedral group $D_{n}$ consists of rotation and reflection transformations, and is thus manifested in the descriptor domain as a combination of rotation and reflection symmetries, $a_{S}\left(t_{i}+t\right)=a_{S}\left(t_{i}+k L / n \pm t \bmod L\right)$ where $i=0, \ldots, n-1$ and $t_{i}$ 's represent the $n$ centers of reflection symmetry 
(note that we do not consider conjugate centers). It can be easily shown that $t_{i}=t_{0}+\frac{L}{2 n} i$. Thus, given one of the centers of reflection symmetry and the order of the symmetry group, we can directly find all other centers.

Table 1 summarizes the characterization of different types of symmetry in the descriptor and its Fourier transform domain.

Table 1. Representation of shape symmetries.

\begin{tabular}{lcc}
\cline { 2 - 3 } & Descriptor domain $a_{S}(t)$ & Fourier domain $\hat{a}_{S}(\omega)$ \\
\hline$C_{n}$ & $L / n$ periodic & discrete with step $2 \pi n / L$ \\
$D_{1}$ & even & linear phase \\
$D_{n}$ & $L / n$ periodic + period even & discrete with step $2 \pi n / L+$ linear phase \\
\hline
\end{tabular}

\subsection{Intrinsic Symmetry Characterization}

Since we assume the descriptors to be local and invariant to rigid transformations, the descriptor sequences of two articulated shapes coincide on the rigid parts. We call this property articulation invariance. Formally, this can be expressed as follows: given an articulation $\mathbf{A}=\left\{\mathbf{R}_{i}, \mathbf{T}_{k}\right\}$ and the intervals $T_{R, i}^{\prime} \subset\left[0, L^{\prime}\right]$ parametrizing the rigid parts $\mathbf{R}_{i} S \subset \mathbf{A} S$ (here $L^{\prime}=\ell(\mathbf{A} S)$ ), we have $a_{S}\left(T_{R, i}\right)=a_{\mathbf{A} S}\left(T_{R, i}^{\prime}\right)$. Explicitly, $T_{R, i}^{\prime}$ are related to $T_{R, i}$ by $T_{R, i}^{\prime}=$ $T_{R, i}+t_{i} \bmod L^{\prime}$ for $t \in T_{R, i}$, where $t_{1}, \ldots, t_{p} \in\left[0, L^{\prime}\right]$ are some offsets.

Combining the articulation invariance relation with extrinsic symmetry characterization, we can characterize intrinsic symmetries. Reversing our notation, assume that $\mathbf{A} S$ is intrinsically symmetric: it is related by an articulation transformation to a shape $S$, which is extrinsically symmetric. Because of articulation invariance, we can consider $S$ instead of $\mathbf{A} S$.

Thus, the intrinsic symmetry case is similar to the extrinsic one, up to insertions into the descriptor sequence at points in which the non-rigid joints are extended and deletions from the descriptor sequence at points in which the nonrigid joints are contracted. If such insertions/deletions are insignificant (i.e., the joints are small compared to the parts sizes), the Fourier domain properties would approximately hold. If the joints are large, we need to explicitly account for insertions/deletions, as described in Sect.4.

\section{Numerical Implementation}

Our analysis so far assumed a continuous curve, but in practice, the shape is sampled at a finite number of points. We assume that our curve $S$ is sampled at $N$ equidistant points (arclength sampling) $S\left(t_{0}\right), \ldots, S\left(t_{N-1}\right)$. The corresponding descriptor is also a discrete sequence $a_{S}\left(t_{0}\right), \ldots, a_{S}\left(t_{N-1}\right)$, denoted here by $\left(a_{0}, a_{1}, \ldots, a_{N-1}\right)$. To simplify the notation, we assume all indices hereinafter modulo $N$. 
The detection and classification of shape symmetry is done by analyzing the discrete descriptor sequence $\left(a_{0}, \ldots, a_{N-1}\right)$. First, we find the symmetry group generators by attempting to detect rotation and reflection symmetry. Next, we classify the symmetry group according to the detected generators. Rotation and reflection symmetry detection is done using one of the following two methods. Fourier analysis, applicable to the extrinsic case and the case of small joints, remains similar to the continuous case. In the case of large joints, we can make use of dynamic programming algorithms employed in text sequence alignment to find the self-similar parts.

\subsection{Fourier Analysis}

In the discrete case, the continuous Fourier transform is replaced by the discrete Fourier transform (DFT), denoted here by $A_{k}=\sum_{m=0}^{N-1} a_{m} e^{-2 \pi \iota k m / N}$, for $k=$ $0, \ldots, N-1$.

Rotation Symmetry. If the shape is $C_{n}$-symmetric, then the discrete descriptor sequence is periodic with period $N / n$, i.e., $a_{m}=a_{m-N / n}$ for $m=0, \ldots, N-1$, and the corresponding DFTs are equal. Since by the shift property the DFT of $a_{m-N / n}$ is $A_{k} e^{-2 \pi \iota k / n}$, we have $A_{k} e^{-2 \pi \iota k / n}=A_{k}$. This leads to the result that $k=m n$ for some integer $m$ for all $k$ where $A_{k} \neq 0$. In other words, the DFT is discrete with step $n$. This gives an easy way to find the symmetry group of the shape by finding the step size of the DFT of its descriptor sequence. To account for the noise, we only look at a neighborhood of the $A_{k}$ with maximum the absolute value.

Reflection Symmetry. In the discrete case, the relation between the center of reflectional symmetry and the phase of the $k$ th element of the DFT becomes $\theta_{k}=\frac{-2 \pi k}{N} t_{i}+2 \pi m_{k} \in[-\pi, \pi]$, for $k=0, \ldots, N-1$. Here, the term $2 \pi m_{k}$ is used to adjust for the phase wrapping and ensures that $\theta_{k}$ lies between $-\pi$ and $\pi$. Using the fact that $t_{i} \leq \frac{N}{2 n}(1+i)$, we get $m_{k} \geq-\frac{1}{2}+\frac{(1+i) k}{2 n}$. In particular, for $k=1$, no wrapping is required (i.e., $m_{1}=0$ ) and we can evaluate the reflection point as $t_{0}=t_{i} \bmod \frac{N}{2 n}$.

Dihedral Symmetry. For the general case of $D_{n}$, the DFT will be discrete as dihedral symmetry also implies $C_{n}$ rotational symmetry. In that case we check only non-zero values for the phase.

\subsection{Dynamic Programming}

Finding symmetries in the Fourier domain is possible in the extrinsic case, or in the intrinsic case when the size of the joints is small. For the case of large joints, we need to account for the joints deformations explicitly. The problem of finding common subsequences in two discrete sequences is very common in text analysis and bioinformatics, where dynamic programming algorithms such as the Smith-Waterman (SWAT) algorithm [29] are used for local sequence alignment. Our case is a particular setting of this problem when we compare a sequence to itself. 

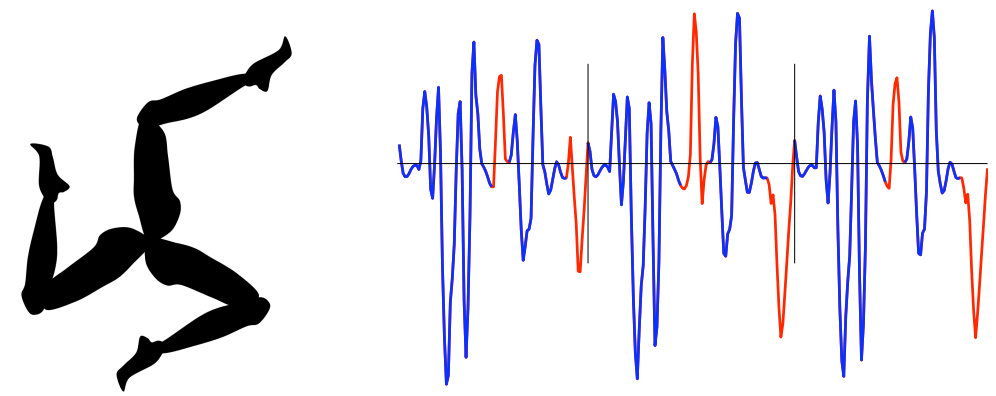

Fig. 1. Rotation symmetry detection using dynamic programming. Left: A $C_{3}$ intrinsically symmetric shape which is extrinsically asymmetric. The size of the joint at the knee is substantial. Right: the corresponding descriptor sequence. Similar parts (blue) correspond to rigid parts; gaps (red) correspond to non-rigid joints.

For each pair of points $a_{i}, a_{j}$ in the sequence, we define a similarity $f\left(a_{i}, a_{j}\right)$. A gap penalty $g\left(a_{i}\right)$ or $g\left(a_{j}\right)$ is defined if one of the points is not matched anywhere and a gap is introduced. We construct an $(N+1) \times(N+1)$ matrix $H$

$$
H_{i j}=\max \left\{H_{i-1, j-1}+f\left(a_{i}, a_{j}\right), H_{i-1, j}+g\left(a_{i}\right), H_{i, j-1}+g\left(a_{j}\right), 0\right\}
$$

and $H_{i 0}=H_{0 j}=0$. Interpreting $H_{i, j}$ as the cost of aligning $a_{i}$ with $a_{j}$, the pair of segments with best similarity is found by going to the maximum element of $H$. The Smith-Waterman algorithm tries to find an alignment with the best cost recursively.

We define the cost function as $f\left(a_{i}, a_{j}\right)=c-F\left(N\left(a_{i}, r\right), N\left(a_{j}, r\right)\right)$ where $c$ is a constant, $N\left(a_{i}, r\right)$ is an $r$ neighborhood of the the point and $F$ is the fraction of points where the two sequences differ by more than a given threshold. Given the length $l$ of the current gap, the gap penalty $g$ is defined as

$$
g_{l}(a)=\left\{\begin{array}{cl}
2 m & \text { if } l=0 \\
m & \text { if } l \leq L \\
M & \text { if } l>L
\end{array}\right.
$$

where $M \gg m$ are constants and $L$ is the maximum anticipated gap length.

Rotation symmetry. To check for rotational symmetry, we match a part of the shape of length $N / n$ with the rest of the shape, for values of $n$ in $2,3, \ldots N / 2$. If we get a match for some $n$, we validate it by dividing the shape into $n$ parts, $S_{1}, S_{2}, \ldots, S_{n}$ of length $L / n$ each, and matching consecutive parts. If validated, we find the principal period of the sequence by looking for periodicity in these parts. It can be easily shown that the alignment corresponding to the principal period serves as a generator of the $C_{n}$ symmetry group of the shape.

Dihedral symmetry. The mappings for rotational symmetry can be evaluated as above. For reflectional symmetry, instead of finding all the mappings, we find 
one mapping and compute the others by composition. For computing the one required mapping, we align the sequence with its reflection. One thing to note here is that the descriptor sequence is defined modulo $N$, which requires to perform circular matching. Following [28], we duplicate one of the sequences for matching.

\subsection{Computational Complexity}

We analyze the complexity of our methods in terms of the number of points $N$ sampled on the shape and the order $n$ of the symmetry group.

Fourier Analysis. Descriptor sequence FFT takes $O(N \log (N))$ time. After that, for reflectional symmetry; taking the arguments(angles) and its unwrapping can be done in linear time. Also, reading the step size can also be done in linear time. So the overall time complexity is $O(N \log (N))$. The time can be further reduced by observing that the complete spectrum is not need for either type of symmetry. The slope of the phase vs frequency curve as well as the step size can be inferred from some of the Fourier coefficients. If we take some constant times $n$ coefficients, the complexity can be reduced to $O(n N)$.

Dynamic Programming. The time complexity of SWAT alignment algorithm is $O(N M)$ for sequences of length $N$ and $M$ respectively. For rotational symmetry, we compare subsequences of length $N / K$ with the complete sequence for values of $\mathrm{K}$ in $2,3, \ldots, n$ where $n$ is the order of the group. This gives the time complexity as $O\left(\log (n) N^{2}\right)$.

\section{Results}

We tested our approach on a set of hand-drawn articulated binary shapes. The shape boundaries were discretized at 300 points. For the local descriptor at point $S(t)$, we used the integral invariant $I(t)=\int_{S}\left\|S(t)-S\left(t^{\prime}\right)\right\| q\left(t, t^{\prime}\right) d t^{\prime}$ with $q\left(t, t^{\prime}\right)=1$ if $\left|t-t^{\prime}\right| \leq 5$ and zero otherwise i.e. we take 10 closest points to the point $S(t)$. Dynamic programming algorithm described in Sect. 4 with parameters $c=0.3, m=0.5, M=1.5$ and $L=7$ was used to find symmetries. Figures $2-4$ show the obtained results. We visualize symmetries by colorings of the shape contour; each coloring denotes the corresponding parts. Thin yellow line denotes the gaps. The figures are best viewed in color print. One can observe that intrinsic symmetry is correctly detected even in the presence of very strong articulations and realistic view artifacts. For comparison, in Fig. 5 we show an example of symmetry detection using the voting method of Loy and Ecklundh [13]. One can observe that strong articulation tamper with this algorithm, bringing in some cases to a failure to detect all the symmetries or an incorrect result.

The proposed approach compares favorably to other methods and has multiple advantages. First, unlike Loy-Eklundh [13] and similar approaches, our method can handle intrinsic symmetries. Second, it allows detecting both reflection and rotation symmetries (unlike Ovsjanikov et al. [20], limited to reflections 

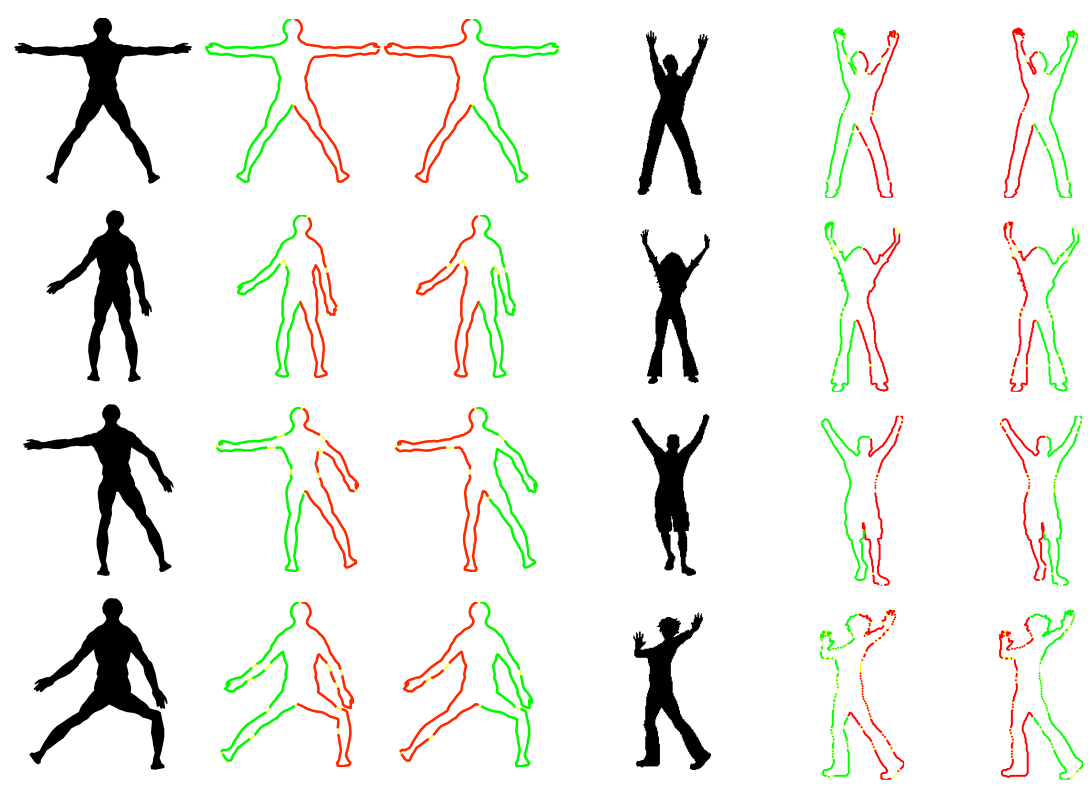

Fig. 2. Detection of bilateral $D_{1}$ symmetry in human figure silhouettes. Each coloring denotes the corresponding parts. Thin lines mark the gaps.

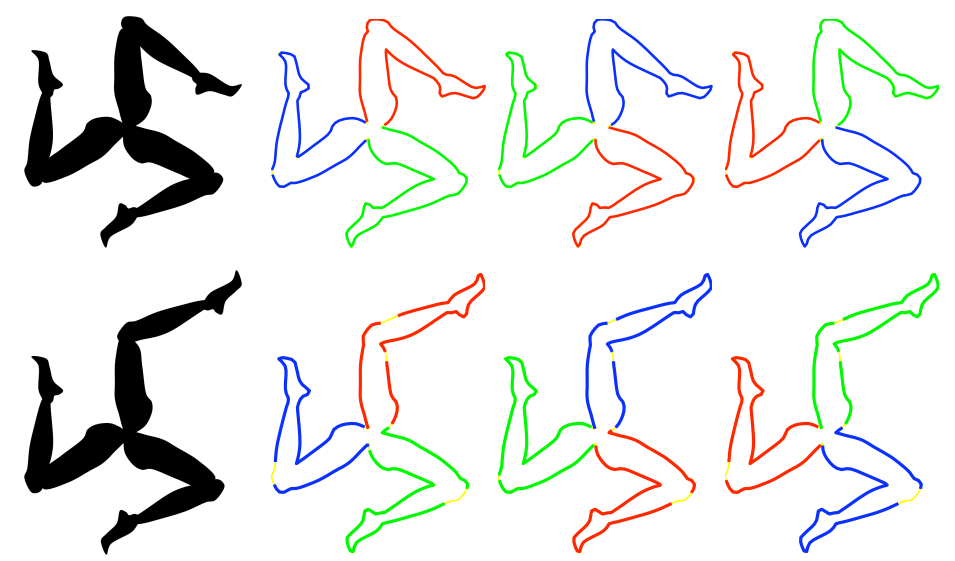

Fig. 3. Detection of $C_{3}$ rotation symmetry in synthetic articulated shapes.

only). Third, our approach guarantees the explicit recovery of all group generators (unlike $[22,20]$ which detect approximate self-isometries that can also be compositions of the generators). Fourth, our dynamic programming (SWAT) approach is capable of handling partial symmetries (as opposed, e.g., to [20]). Fifth, our approach has guaranteed low computational complexity ( $N$ for Fourier analysis or $N^{2}$ for SWAT, where $N$ is the number of samples), compared to the theoretically worst-case $N$ ! of [22] (though in practice much lower). Finally, our 

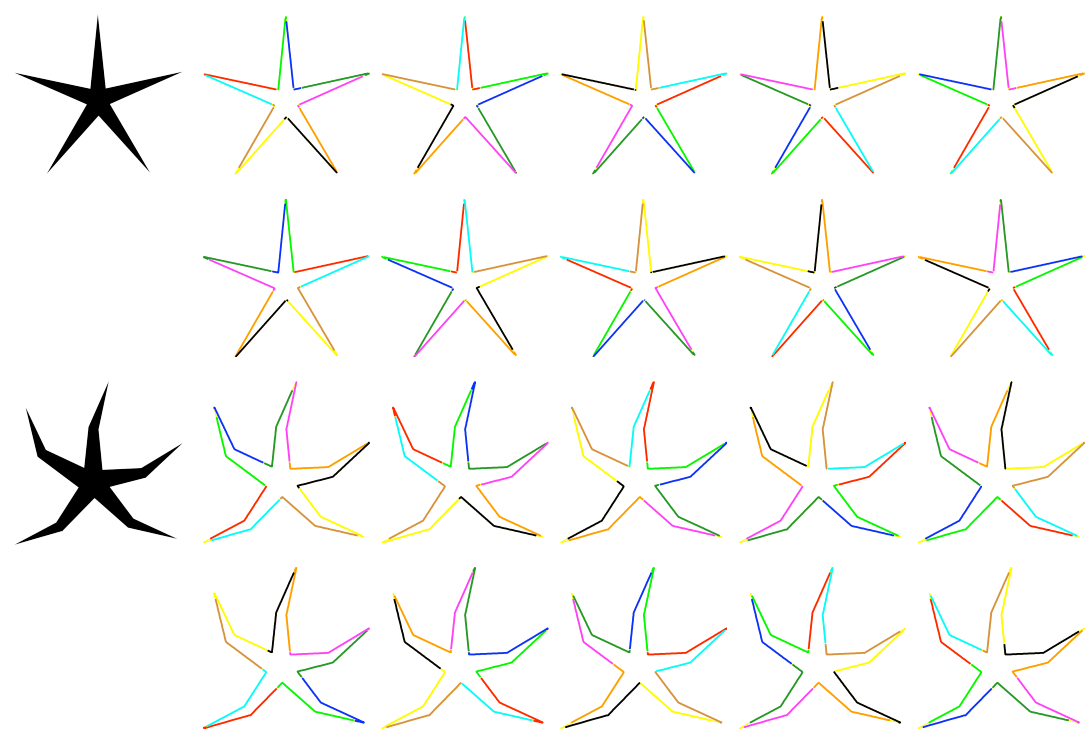

Fig. 4. Detection of $D_{5}$ dihedral symmetry in synthetic articulated shapes (first row: extrinsic, second through fourth rows: intrinsic).

approach is straightforwardly generalizable to other classes of invariance (e.g. affine) by appropriate choice of the descriptor. The comparison is summarized in Table 2 .
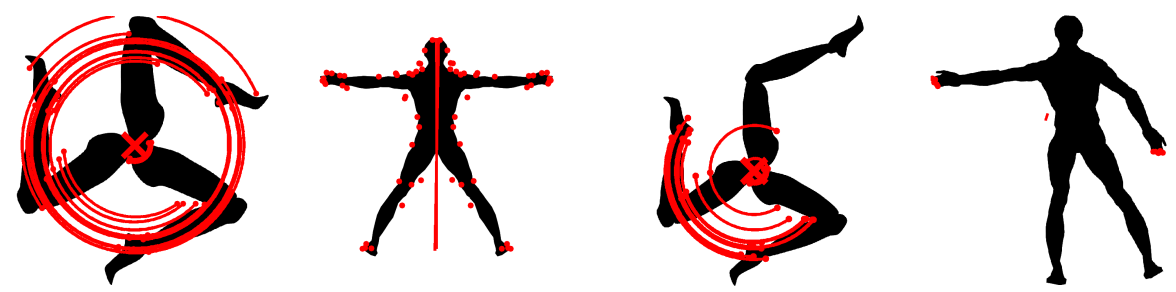

Fig. 5. Performance of the Loy-Eklundh [13] symmetry detection algorithm on our shapes. Left to right: correctly detected rotational $C_{3}$ symmetry and bilateral $D_{1}$ symmetry, partial $C_{3}$ symmetry detection (only one rotation), and incorrect bilateral symmetry detection.

\section{Conclusions}

We presented an approach for detection of symmetries in articulated 2D shapes, based on representing the shape contours using invariant local descriptors and 
Table 2. Comparison of methods for symmetry detection. Note: ${ }^{a}$ Extended to $3 \mathrm{D}$ shapes case in [21]. ${ }^{b}$ Extension to $2 \mathrm{D}$ is straightforward. ${ }^{c}$ Extended to intrinsic symmetries in plane-like 3D shapes in [16]. ${ }^{d}$ Partial symmetries addressed in [23]. ${ }^{e}$ Theoretical worst case that guarantees global optimality. ${ }^{f}$ Using fast approximate nearest neighbor search; there is additional factor $d^{3}$ depending on the number of eigenfunctions used, $d$. ${ }^{g}$ Computing only the first few frequencies as explained in Sect. $4 .{ }^{h}$ Invariance depends on the descriptor. ${ }^{i}$ Invariance depends on the metric.

\begin{tabular}{lccccc} 
& {$[13]$} & {$[22]$} & {$[20]$} & FA & SWAT \\
\hline 2D/3D & Both $^{(a)}$ & Both $^{(b)}$ & Both $^{(b)}$ & $2 \mathrm{D}$ & $2 \mathrm{D}$ \\
Extrinsic/Intrinsic & Ext $^{(c)}$ & Both & Both & Both & Both \\
Reflection/Rotation & Both & Both & Ref & Both & Both \\
Topological noise & + & + & + & - & - \\
Partial symmetry & + & $+{ }^{(d)}$ & - & - & + \\
Complexity & $N^{2}$ & $N !^{(e)}$ & $N \log N^{(f)}$ & $N^{(g)}$ & $N^{2}$ \\
General invariance & $+^{(h)}$ & $+^{(i)}$ & - & $+^{(h)}$ & $+^{(h)}$ \\
\hline
\end{tabular}

characterizing shape symmetries as patterns in the associated descriptor sequences. Such patterns are preserved under non-rigid deformations and can be efficiently detected using Fourier analysis or dynamic programming sequence alignment algorithms. The main limitation of our approach is the assumption of simple closed curves, which implies that the underlying shape has no disconnected components and has simple topology.

\section{References}

1. Alt, H., Mehlhorn, K., Wagener, H., Welzl, E.: Congruence, similarity, and symmetries of geometric objects. Discrete Comput. Geom. 3, 237-256 (1988)

2. Atallah, M.J.: On symmetry detection. IEEE Trans. Computers c-34(7) (Jul 1985)

3. Bokeloh, M., Berner, A., Wand, M., Seidel, H., Schilling, A.: Symmetry detection using line features. In: Computer Graphics Forum (Special Issue of Eurographics). vol. 28 , pp. $697-706$ (2009)

4. Bruckstein, A., Shaked, D.: Crazy Cuts: Dissecting Planar Shapes into Two Identical Parts. Mathematics of Surfaces XIII pp. 75-89 (2009)

5. Cheung, K., Ip, H.: Symmetry detection using complex moments. In: Proc. International Conference on Pattern Recognition (ICPR). vol. 2, pp. 1473-1475 (1998)

6. Cornelius, H., Loy, G.: Detecting rotational symmetry under affine projection. In: Proc. International Conference on Pattern Recognition (ICPR). vol. 2, pp. 292-295 (2006)

7. Derrode, S., Ghorbel, F.: Shape analysis and symmetry detection in gray-level objects using the analytical fourier-mellin representation. Signal Processing 84(1), 25-39 (2004)

8. Frenkel, M., Basri, R.: Curve matching using the fast marching method. In: Energy Minimization Methods in Computer Vision and Pattern Recognition. pp. 35-51 (2003)

9. Gofman, Y., Kiryati, N.: Detecting symmetry in grey level images: The global optimization approach. In: Proc. International Conference on Pattern Recognition (ICPR). pp. 951-956 (1996) 
10. Kazhdan, M., Chazelle, B., Dobkin, D., Funkhouser, T., Rusinkiewicz, S.: A reflective symmetry descriptor for 3D models. Algorithmica 38(1), 201-225 (2003)

11. Ling, H., Jacobs, D.: Shape classification using the inner-distance. Trans. PAMI 29(2), 286-299 (2007)

12. Liu, Y., Hel-Or, H., Kaplan, C.S., van Gool, L.: Computational symmetry in computer vision and computer graphics. Foundations and Trends in Computer Graphics and Vision 5(1-2), 1-195 (2010)

13. Loy, G., Eklundth, J.: Detecting symmetry and symmetric constellations of features. In: Proc. CVPR. vol. 2, pp. 508-521 (2006)

14. Manay, S., Hong, B.W., Yezzi, A.J., Soatto, S.: Integral invariant signatures. In: Proc. ECCV (2004)

15. Marola, G.: On the detection of axes of symmetry of symmetric and almost symmetric planner images. Trans. PAMI 11(1) (Jan 1989)

16. Mitra, N.J., Bronstein, A.M., Bronstein, M.M.: Intrinsic Regularity Detection in 3D Geometry. In: Proc. ECCV (2010)

17. Mitra, N.J., Guibas, L.J., Pauly, M.: Partial and approximate symmetry detection for 3D geometry. ACM Trans. Graphics (TOG) 25(3), 568 (2006)

18. Mitra, N.J., Guibas, L.J., Pauly, M.: Symmetrization. In: Proc. SIGGRAPH (2007)

19. Natale, F.G.B.D., Giusto, D.D., Maccioni, F.: A symmetry-based approach to facial features extraction. In: Proc. International Conference on Digital Signal Processing Proceedings (ICDSP). vol. 2, pp. 521-525 (1997)

20. Ovsjanikov, M., Sun, J., Guibas, L.J.: Global intrinsic symmetries of shapes. In: Proc. SGP. vol. 27 (2008)

21. Pauly, M., Mitra, N.J., Wallner, J., Pottmann, H., Guibas, L.J.: Discovering structural regularity in 3D geometry. ACM Trans. Graphics (TOG) 27(3), 43-43 (2008)

22. Raviv, D., Bronstein, A.M., Bronstein, M.M., Kimmel, R.: Symmetries of non-rigid shapes. In: Proc. Non-rigid Registration and Tracking (NRTL) (2007)

23. Raviv, D., Bronstein, A.M., Bronstein, M.M., Kimmel, R.: Full and partial symmetries of non-rigid shapes. IJCV 89(1), 18-39 (2010)

24. Riklin-Raviv, T., Kiryati, N., Sochen, N.: Segmentation by level sets and symmetry. In: Proc. CVPR (2006)

25. Shimshoni, I., Moses, Y., Lindernbaum, M.: Shape reconstruction of 3D bilaterally symmetric surfaces. IJCV 39(2), 97-110 (2000)

26. Sorkine, O., Lévy, B., Kim, V.G., Lipman, Y., Chen, X., Funkhouser, T.: Möbius Transformations For Global Intrinsic Symmetry Analysis (2010)

27. Sun, C., Sherrah, J.: 3D symmetry detection using the extended gaussian image. Trans. PAMI 19(2), 164-168 (1997)

28. Uliel, S., Fliess, A., Amiry, A.: A simple algorithm for detecting circular permutations in proteins. Bioinformatics 15, 15-11 (1999)

29. Waterman, M.S., Smith, T.F.: Identification of common molecular subsequences. J. Mol. Biol. 147, 195-197 (1981)

30. Weyl, H.: Symmetry. Princeton University Press (1983)

31. Wolter, J.D., Woo, T.C., Volz, R.A.: Optimal algorithms for symmetry detection in two and three dimensions. The Visual Computer 1, 37-48 (1985)

32. Xu, K., Zhang, H., Tagliasacchi, A., Liu, L., Li, G., Meng, M., Xiong, Y.: Partial intrinsic reflectional symmetry of 3D shapes. ACM Trans. Graphics (TOG) 28(5), 3 (2009)

33. Zabrodsky, H., Peleg, S., Avnir, D.: Symmetry as a continuous feature. Trans. PAMI 17(12), 1154-1166 (1995) 